\title{
Biografía de Sabina Spielrein (1885-1942): una historia de los primeros años del psicoanálisis
}

RESUMEN: El tratamiento psicoanalítico de la histeria y el nacimiento de la psicología infantil a través de la biografía de la psiquiatra rusa Sabina Spielrein.

PALABRAS CLAVE: Sabina Spielrein, hospital Burghölzli, psicoanálisis, psicología infantil.
SUMMARY: The psychoanalytic treatment of hysteria and the birth of children psychology through biography of the Russian psychiatrist Sabina Spielrein.

KEY WORDS: Sabina Spielrein, Burghölzli Hospital, psychoanalysis, child's psychology.

Nos proponemos exponer la vida de Sabina Spielrein, psiquiatra y psicoanalista de principios del siglo XX, cuya biografía es interesante por dos motivos: primero porque su contacto con ambas disciplinas fue como paciente psiquiátrica grave. Estuvo ingresada en el hospital Burghölzli, en Zúrich, a cargo del entonces psiquiatra de la institución Carl Gustav Jung y han aparecido recientemente los documentos de la historia clínica traducida al inglés. El segundo motivo se refiere al contexto histórico y científico en el que desarrolló su obra: durante el primer tercio del siglo XX, importante en la historia de la psiquiatría por ser el momento en que apareció el concepto de esquizofrenia bleuleriano, se desarrollaron las teorías del psicoanálisis, entonces una disciplina naciente, surgieron las primeras Asociaciones Psicoanalíticas en Europa y aparecieron los primeros textos de psicología infantil y sobre el desarrollo del niño, todo ello aspectos en los que colaboró Sabina Spielrein en mayor o menor medida.

\section{Breve reseña de su infancia}

Sabina Spielrein nació en Rostov el 7 de Noviembre de 1885. Fue la hija mayor de un matrimonio judío de clase social alta que tendría cuatro hijos más. A los cinco años comenzó sus estudios interna en Varsovia, y a los ocho volvió a Rostov a continuarlos en su ciudad natal (1). La describen como una niña difícil, castigada frecuentemente, con un interés sexual muy precoz y nada reprimido (2), una imaginación desbordante (3) y una inteligencia precoz (4). Obtuvo unos resultados académicos brillantes durante su etapa de formación y era políglota (3).

Su padre era comerciante y su madre odontóloga (2). En la descripción que Jung refleja en la historia clínica de Sabina Spielrein sobre ellos los define a 
ambos como histéricos: el padre es nervioso, colérico, utiliza con frecuencia los castigos físicos (5) y a veces con una connotación vejatoria y humillante (4), es manipulador, tiránico e insultante. En la historia clínica se relata un episodio en el que el hombre amenaza con el suicidio cuando su hija Sabina, con dieciséis años, le refiere ideas de independencia (quiere conocer otras personas fuera de la familia), ejemplar para hacernos una idea de su forma de ser y la relación que mantiene con su familia (4). Su madre está descrita por Jung como agitada, con comportamiento infantil. Es una mujer seductora, que parece comportarse con su hija como una rival en ocasiones (1). Por otro lado sus tres hermanos (tuvo una cuarta hermana que murió a los seis años, cuando Sabina Spielrein tenía dieciséis) son nerviosos y somatizadores, a juicio de Jung (sufrían tics, molestias gástricas) (5). Los tres fueron hombres inteligentes, de formación universitaria, e incluso uno de ellos colaboró con Sabina Spielrein en uno de sus trabajos publicados. Serían fusilados en distintos momentos de la década de los treinta por el gobierno estalinista (6). La relación de los padres con sus cuatro hijos está definida en la historia clínica de Sabina Spielrein como «tumultuosa» y «sadomasoquista» (7).

\section{La enfermedad: el contacto con el psicoanálisis}

La información sobre la enfermedad de Sabina Spielrein proviene fundamentalmente de los archivos del hospital Burghölzli, del diario de Sabina Spielrein, que aparece publicado íntegramente en el libro de Aldo Carotenuto (8) y de una carta de Jung a Freud exponiéndole el caso. Además Jung presentó este caso clínico en el I Congreso de Psiquiatría y Neurología en Ámsterdam en 1907, como reflejo de la teoría freudiana de la histeria (8).

Sabina Spielrein comenzó a presentar síntomas de un trastorno mental a la edad de cuatro años. En ese momento su comportamiento consistía en retener las heces, a veces durante dos semanas, ocluyendo en ocasiones el ano con el talón. Este comportamiento le provocaba un placer evidente y lo manifestaba en público (8). Asimismo entre el tercer y el cuarto año de vida le impresionó un castigo corporal, una paliza que le inflingió su padre a su hermano. Éste estaba desnudo y Sabina Spielrein al verlo pensó, de forma intrusiva, que ella había defecado en la mano de su padre. A los siete años cambió la conducta relativa a la defecación por la masturbación compulsiva (8), manifestando ideas obsesivas de índole sexual que tenían como desencadenante todo lo relacionado con la comida y con los castigos corporales o las situaciones violentas, sobre todo si provenían de su padre. Llegó a no poder comer en público y cuando la golpeaban o golpeaban a sus hermanos debía masturbarse (9). Hasta este momento lo llamativo en su historia son las conductas compulsivas y las ideas intrusivas, y los temas a los que están aso- 
HISTORIA DE LA PSIQUIATRÍA

ciadas. A los dieciséis años la clínica cambia y su estado empeoró: presentaba continuos cambios de humor de la risa al llanto, comportamiento impulsivo, fugas sin objeto, actings parasuicidas (3). Ella, en comentarios posteriores de su diario, recuerda la muerte de su hermana como uno de los desencadenantes de su empeoramiento (8). Primero estuvo ingresada en dos clínicas suizas; en una de ellas le dieron como tratamiento electrochoques, para acabar ingresando en agosto de 1904 en Burghölzli, Zúrich, hasta Junio de 1905 (3; 5).

Burghölzli fue el centro de hospitalización psiquiátrica para pacientes con patología aguda de la ciudad de Zúrich desde 1870. Existía otro centro en las afueras de Zúrich para pacientes crónicos, irrecuperables $(4 ; 10)$. Burghölzli era un centro de 400 camas, a pesar de que, de forma habitual, tenía mucho mayor número de pacientes ingresados. Estaba asociado a la Universidad de Zúrich. Su lema, desde el diseño de la clínica por parte de Wilhelm Griesinger, era: «Las enfermedades de la mente son enfermedades del cerebro». Pero a pesar que esto podría suponer un concepto de la psiquiatría poco permeable a las teorías psicoanalíticas, lo cierto es que existió un interés por la ciencia en ciernes que era por aquel entonces la teoría psicoanalítica. Este interés se ve reflejado en los trabajos de Forel con la hipnosis y la sugestión y la creación de una revista en 1892 sobre este tema, la Revista de Hipnotismo y Terapia de Sugestión, la adopción por Bleuler del origen psicógeno de los síntomas secundarios de la Demencia Precoz (teorizó que los síntomas secundarios eran representaciones de deseos inconscientes, y un mecanismo de defensa del «yo» ante la enfermedad) y los trabajos sobre asociaciones verbales que llevaron a cabo Jung y Riklin en el centro, en los que participaría Sabina Spielrein, en un primer momento cuando estaba ingresada y posteriormente como estudiante de medicina (4).

La evolución de Sabina Spielrein durante el ingreso en Burghölzli tuvo altibajos: presentó conductas negativistas, mutismo, agresividad e irritabilidad y comportamiento provocador con el personal del centro. También se reflejan oscilaciones frecuentes de su estado de ánimo, ansiedad y sensaciones de despersonalización (4). La historia clínica refleja perfectamente las dificultades de su manejo en el hospital. Los cambios de humor se exacerbaban cuando recibía noticias de su familia, visitas o cartas, que suponían por lo general un empeoramiento en la evolución. También empeoraba cuando Jung, su psiquiatra, se ausentaba del centro y debía asumir su asistencia Bleuler (parece que conseguir una buena alianza terapéutica con ella fue un proceso complicado y lento). Tenía pesadillas en forma de asaltos de personas o sombras, durante la noche y en cama, que le provocaban miedo y sensación de suciedad (5). Pero finalmente la mejoría fue tal que, ingresada todavía, en abril de 1905 se matriculó en la facultad de medicina de Zúrich con la idea de ser psiquiatra, y se incorporó como ayudante en los trabajos de Jung y Riklin $(3 ; 8)$. 
Sabina Spielrein fue la primera paciente tratada por Jung con el «método analítico». Entrecomillamos el método analítico debido a que fue en verdad un tratamiento heterodoxo, aplicado en un momento en que la técnica psicoanalítica distaba mucho de estar consensuada y depurada. Consistió en un alejamiento radical de su familia de origen, hasta el punto de impedir el que uno de sus hermanos estudiase en Zúrich, un manejo del personal sustentador, tranquilizador, previniendo los acting-outs y haciéndola sentir aceptada y autosuficiente y técnicas sugestivas, capaces de reforzar su super ego (7).

El diagnóstico reflejado en su historia clínica tras la entrevista de ingreso y al alta fue el mismo: histeria. Jung escribe en su historia clínica que desde la infancia se reconocen en la paciente comportamientos histéricos. Incluso en la entrevista del ingreso, en plena crisis de agitación y alternancia de risas y llanto, describe «miradas seductoras» (4). Contextualizando un poco el diagnóstico de Sabina Spielrein, recordemos que en su primera definición de la histeria, en 1895, Freud escribe que los síntomas están específicamente vinculados a traumas de seducción o sexuales y que a partir de 1897 consideraría en cambio su etiología como debida a eventos puramente psíquicos (7). Jung por su parte considera en 1906 la histeria como una condición morbosa en la que los afectos son extremistas y hacen al sujeto víctima de ellos (7). Algunos autores consideran en cambio que es posible que el diagnóstico fuese verdaderamente un trastorno por estrés postraumático secundario a abusos sexuales, y que las pesadillas parecen reviviscencias de esos abusos (4). Sea cual sea el diagnóstico ilustran parcialmente la personalidad de Sabina Spielrein dos frases, una sacada de su diario: «ningún dolor me es demasiado insufrible y ningún sacrificio demasiado grande como para impedirme cumplir con mi destino sagrado»(2) y aquella con la que la describió Freud, «como perteneciente al tipo salvadora o sacrificada» (11).

Ya de alta hospitalaria y estudiante de medicina en la Universidad de Zúrich con muy buenos resultados académicos, continuó siendo paciente ambulatoria de Jung hasta 1909 (10).

\section{La importancia de la relación entre la paciente y su psicoanalista}

La relación entre Sabina Spielrein y Jung, entre 1904 y 1909, es importante para la historia del psicoanálisis por varios motivos. En primer lugar si fue la primera paciente tratada por Jung con el método analítico, además la relación entre ambos pasó de ser la de médico-paciente a ser una relación amorosa, y es la primera vez que esto ocurre de forma documentada (12). Al menos fue así desde principios de 1908 hasta Marzo de 1909, en que la relación se hizo pública y Sabina Spielrein dejó de ser paciente en análisis de Jung. También, en marzo de 1909, Jung presenta la renuncia al cargo que ocupaba como psiquiatra del hospital Burghölzli (8). 
HISTORIA DE LA PSIQUIATRÍA

En segundo lugar porque supuso para Jung el inicio de una relación personal con Freud, desde el momento en que aquél le relató el caso de Sabina Spielrein, en octubre de 1906, solicitándole algo parecido a una supervisión (8; 13). Freud mantuvo contacto con Jung y con la propia Sabina Spielrein, que le escribió por primera vez en el momento en que la relación amorosa existente entre ella y Jung fue de dominio público. La postura de Freud al principio, ante la relación entre ambos y su repercusión, fue la justificación de la conducta de Jung y la solicitud a Sabina Spielrein de que «reprimiese sus sentimientos hacia Jung». La relación que Freud quería mantener con Jung en ese momento era especial: lo consideraba el sucesor que haría posible por un lado la expansión del psicoanálisis fuera del ámbito judío (6) y, por otro, la continuación de su trabajo aplicando las teorías psicoanalíticas en el campo de las psicosis, como él hasta el momento había hecho con las neurosis, dada la experiencia de Jung adquirida en Burghölzli con Bleuler (12).

Pero al mismo tiempo, y en tercer lugar, muchos autores creen que la relación entre Jung y Sabina Spielrein también fue el motivo de la ruptura entre los dos; Jung y Freud, argumentando que la dificultad de Jung para conceder un papel principal a la sexualidad en la etiología de las neurosis provenía de sus dificultades personales para manejarla en el contexto analítico, y que la relación entre Jung y Sabina Spielrein es un ejemplo de esta dificultad, como también ocurriría posteriormente con Toni Wolf $(8 ; 13)$. También sabemos que en 1913, cuando la ruptura entre Jung y Freud era obvia, Freud escribió que la opinión que le merecía el comportamiento de Jung hacia Sabina Spielrein había cambiado, desde esa postura previa justificadora a definirlo como detestable $(8 ; 12)$.

En cuarto lugar, Freud, tras el conocimiento de este caso y ya en el verano de 1909, pretendía escribir una serie de técnicas y reglas sobre la práctica del psicoanálisis para sus discípulos más cercanos. En la correspondencia que mantiene con Jung respecto a su relación con Sabina Spielrein, utiliza por primera vez el término «contratransferencia», exhortándole a aprender de las dificultades para desplazar sus sentimientos (12). En el II Congreso Internacional de Psicoanálisis en 1910, en Nüremberg, estructuró sus ideas sobre este aspecto de la técnica psicoanalítica hablando de la necesidad de dominar y analizar sus propios sentimientos por parte del analista, y la de realizar el análisis en abstinencia por parte del analizado $(13 ; 14)$.

\section{La carrera profesional de Sabina Spielrein}

La carrera profesional de Sabina Spielrein comenzó ligada a la escuela de Zúrich representada por Bleuler y Jung. Recordemos que comenzó a trabajar en los experimentos sobre asociaciones verbales cuando estaba ingresada en Burghölzli, y que continuaría estos trabajos mientras estudiaba en la Facultad de 
medicina de Zúrich. En 1911 se licenció con la lectura de su tesis El contenido psicológico de un caso de esquizofrenia, bajo la dirección de Bleuler y en colaboración con Jung. En este trabajo Sabina Spielrein utiliza los experimentos sobre asociaciones verbales para «descifrar» el lenguaje desorganizado y delirante de una paciente con esquizofrenia, comparando los mecanismos del pensamiento de la paciente con la mitología (8). Este trabajo tiene además la particularidad de que constituye el primer texto académico en que se utiliza por primera vez el término esquizofrenia (15). Muchos autores, entre otros Carotenuto y Bettelheim consideran que durante sus años en Zúrich la relación con Jung fue lo que indujo a éste a estructurar las teorías de Ánimus-ánima (que sostiene la existencia de una parte del inconsciente del hombre que personifica la naturaleza femenina y de una naturaleza masculina en el inconsciente de la mujer) y la Sombra (que se refiere a la existencia en el inconsciente de una personalidad oculta, reprimida y que suele tener un valor inferior y culpable. Esta personalidad parcial es el resultado de la represión de las cualidades que conceptuamos como inaceptables y no queremos reconocer en nosotros mismos), con Sabina Spielrein en calidad de colaboradora, inspiradora o coautora, como reconoció Jung en una carta y aparece en los diarios de ella $(2 ; 8)$.

Desde octubre de 1911 a marzo de 1912 vive en Viena, donde tomó contacto personal con Freud, al que ya conocía por la correspondencia mantenida al final de su relación amorosa con Jung. Ingresa en la Asociación Psicoanalítica vienesa y lee en ella su segundo trabajo más importante; La destrucción como causa del nacimiento, ante Otto Rank, Víctor Tausk, Wilhem Stekel, Paul Federn, Hanns Sachs y el propio Freud a finales de noviembre de 1911. Este trabajo de Sabina Spielrein, que se publicaría un año después, para algunos autores constituye el germen de lo que posteriormente será la «pulsión de muerte» freudiana. El mismo Freud reconoce, en una nota al pie de página de su libro, Más allá del principio de placer, donde expone su teoría sobre la pulsión de muerte, la anticipación que tuvo Sabina en sus ideas $(3 ; 13)$. En este texto $S$. Spielrein desarrolla la teoría de que en el propio instinto de conservación, que se pone de manifiesto en el deseo sexual, existe una tendencia, o instinto de destrucción. Hace una revisión sobre el conflicto fundamental del psiquismo entre las pulsiones sexuales y las pulsiones de autoconservación, para proponer el conflicto entre la vida y la muerte (13). Pero no todos los autores están de acuerdo en que este trabajo sobre la pulsión de muerte sea un predecesor de las teorías freudianas: en un artículo de Vallejo Orellana y Sánchez-Barranco Ruiz sobre Sabina Spielrein explican que desde su punto de vista el trabajo de Sabina Spielrein sería más bien una teoría sobre la represión: el instinto sexual estaría enfrentado con el instinto de conservación. El instinto de conservación tiene como objetivo mantener la individualidad del yo frente a cambios no deseados, mientras que el inconsciente, sobre el que Sabina Spielrein tiene una concepción colectiva y no individual como discípula de Jung que fue, tendría como objetivo la conservación de la espe- 
HISTORIA DE LA PSIQUIATRÍA

cie por encima del yo individual. Por eso el instinto sexual, conservador de la especie, actuaría contra el yo, y éste vive tal instinto como una amenaza. Por ello la represión actúa sobre la sexualidad específicamente (3). En este artículo es (de todos los trabajos sobre Sabina Spielrein) donde más extensamente se comentan sus obras.

A partir de aquí comenzó un periplo por distintas capitales europeas practicando el psicoanálisis y publicando artículos sobre psicología infantil, que centró sus investigaciones desde 1912 (13). En Berlín, en 1912, se casó con Paul Scheftel, con el que tuvo dos hijas, Renata y Eva, y del que se separó en 1915 (11).

Desde 1912 hasta 1926, año de su vuelta a Rostov, vivió y trabajó en Berlín, Munich, Lausana, Ginebra y Moscú (1). En Berlín perteneció a la Asociación Psicoanalítica (3). En Ginebra organizó la Sociedad Psicoanalítica de la ciudad (11) y fue profesora de psicoanálisis en el Instituto Rosseau, siendo analista de Claparede, Charles Odier, Charles Bally y Jean Piaget. Con estos dos últimos publicó trabajos en común (13). En Moscú trabajó con Vera Schmidt, Vigotzky y Luria (13). Le ofrecieron una cátedra en la Universidad de Moscú y la dirección de una clínica psicoanalítica infantil (13). Una parte importante de su vida, la que abarca desde el traslado de Viena hasta poco antes de su muerte, la dedicó a la investigación y la asistencia infantil. Sobre todo escribió sobre el desarrollo temprano en el niño, y especialmente sobre el desarrollo del lenguaje, registrando las observaciones de su primera hija, Renata, y comparándolas con las de otros escritores. Llegó a conclusiones, como el concepto del lenguaje como una capacidad innata, aunque necesitada de estímulos para desarrollarse, que encontraremos en las obras de otros autores en fechas posteriores. Sabina Spielrein escribió durante toda su carrera un total de 29 publicaciones sobre psicología infantil, lingüística y desarrollo del lenguaje y mantuvo una correspondencia fluida con Jung y Freud (8).

Dado que el psicoanálisis no seguía las directrices del gobierno bolchevique, en 1929 aparecieron fuertes restricciones a la práctica analítica y se disuelve la Sociedad Psicoanalítica de Moscú. Acabó por prohibirse su práctica en 1936 por parte del gobierno estalinista de la Unión Soviética (13). Sabina Spielrein se trasladó a Rostov en 1926, dedicándose a la constitución y dirección de un hogar para lactantes y niños (13). Se pierde su pista en 1937, pues a partir de esa fecha no acude a ningún congreso ni publica ningún trabajo. En 1942 fue fusilada en la Sinagoga de Rostov por soldados alemanes del ejercito nazi, del frente del este, durante la Segunda Guerra Mundial (1).

\section{Conclusiones}

Sabina Spielrein, por su historia personal y por su producción científica, es un personaje de importancia en la historia del psicoanálisis, que, como ejemplo de 
un olvido generalizado, las biografías y memorias de Jung y Freud respectivamente no reconocieron (15). Por su historia personal es la primera paciente a la que Jung trata con el método psicoanalítico cuando éste empezaba. Ella es el motivo del primer acercamiento personal entre Jung y Freud y posiblemente también en mayor o menor medida el motivo personal de su ruptura.

Su caso es también uno de los primeros encontronazos documentados con las dificultades transferenciales y uno de los motivos que lleva a Freud a la publicación de los métodos del análisis (12) para ayudar a los analistas a resolver esas dificultades.

Respecto a su obra, se trata de una de las primeras autoras psicoanalíticas (3). Está involucrada como inspiradora o coautora en las teorías jungianas de Ánima-ánimus y la Sombra (8). Publica el primer trabajo académico en que se utiliza el neologismo esquizofrenia y uno de los primeros trabajos de la escuela de Zúrich en adoptar la unión entre psicología y esquizofrenia (15). Con sus obras se convierte en una pionera en el desarrollo de la psicología infantil y es la primera autora en vincular las teorías freudianas con el lenguaje (11). Esto a pesar de ser recordada principalmente por su relación amorosa con Jung, las indiscreciones cometidas por éste y su posición en la relación entre Jung y Freud. Fue famosa más por la asociación de su nombre con el de ambos que por su propio mérito (11).

\section{BIBLIOGRAFÍA}

(1) Alnaes, K., La verdadera historia de Sabina Spielrein, Madrid, Siruela, 2004.

(2) Volnovich, J. C., «Sabina Spielrein: expropiación intelectual en la historia del psicoanálisis», 2002, http://psicomundo.com/foros/genero/sabina.htm.

(3) Vallejo Orellana, R.; SÁnchez-Barranco Ruiz, A., «Sabina Spielrein, la primera mujer que enriqueció la teoría psicoanalítica», Revista de la AEN, 2003, 85, pp. 107-122.

(4) Graf-Nold, A., «The Zürich Scholl of Psychiatry in Theory and Practice. Sabina Spielrein's Treatment at the Burghölzli Clinic in Zürich», J. Anal. Psychol., 2001, 46, pp. 73-104.

(5) Steffens, D.; Wharton, B., «Burghölzli Hospital Records of Sabina Spielrein», J. Anal. Psychol., 2001, 46, pp. 15-42.

(6) Gez, B., «La mujer que «inventó» la pulsión de muerte», Fundación Descartes 2004, http://www.descartes.org.ar/modulos-cuerpo-lamujer.htm.

(7) Covigton, C., «Comments on the Burghölzli Hospital Records of Sabina Spielrein», J. Anal. Psychol., 2001, 46, pp. 105-116.

(8) Carotenuto, A., Una secreta simetría. Sabina Spielrein entre Freud y Jung, Barcelona, Gedisa, 1984.

(9) Minder, B., «Sabina Spielrein. Jung's Patient at the Burghölzli», J. Anal. Psychol., 2001, 46, pp. 43-66. 
HISTORIA DE LA PSIQUIATRÍA

(10) Minder, B., «A Document Jung to Freud 1905: a Report on Sabina Spielrein», J. Anal. Psychol., 2001, 46, pp. 67-72.

(11) Cifali, M., «Sabina Spielrein, Woman Psychoanalyst: Another Picture», J. Anal. Psychol., 2001, 46, pp. 129-138.

(12) Marcos-Turnbull, R., «Diez francos la consulta», http://www.mecayoelveinte.com/ anteriores/pdf/10-rodolfo\%20Marcos.pdf.

(13) GarCía Ávila, A., «Un art amatoria: la historia de Sabina Spielrein en los avatares de la transferencia», Textura, 2006, 1, pp. 107-126. http://www.textura.clinicaanemos.net/textura-01pdf.

(14) Vartuli, M. A., «Un beso o diez francos», Revista Internacional de Psicología, 2002, 1, enero-junio, http://www.español.geocities.com/aguilera99/volumen2.htm.

(15) GarRabÉ, J., «Sabina Spielrein: el nacimiento de la esquizofrenia (1906-1912)», Salud Mental, 1996, 4, pp. 43-51.

Los artículos referenciados como los números 4, 5, 7, 9 y 10, además de incluirse en la revista citada, aparecen compilados en Covington, C.; Wharton, B. (eds.), Sabina Spielrein: Forgotten Pioneer of Psychoanalysis, Nueva York, Brunner-Routledge, 2003, donde aparece la historia clínica de Sabina Spielrein traducida al inglés de los documentos originales del Hospital Burghölzli.

* Marina Fuentes Barco; Belén Martínez Alonso; Sergio Piñeiro García. Servicio de Psiquiatría de Vigo; Tiburcio Angosto Saura, jefe de Servicio de Psiquiatría de Vigo. Organizador de las «Jornadas de Clásicos en Psiquiatría. Vigo».

Correspondencia: Marina Fuentes Barco. Servicio de Psiquiatría del Complejo Hospitalario Universitario de Vigo. Hospital Nicolás Peña. Calle Camelias, $n^{\circ}$ 109. 36211. Vigo: mcfuentesbarco@hotmail.com

** Fecha de recepción: 24-V-2007 (aceptado el 10-IX-2007). 